Research Article

\title{
Exploration on Robustness of Exponentially Global Stability of Recurrent Neural Networks with Neutral Terms and Generalized Piecewise Constant Arguments
}

\author{
Wenxiao Si $(\mathbb{D}$, Tao Xie $\mathbb{D}$, and Biwen $\mathrm{Li}(\mathbb{D}$ \\ College of Mathematics and Statistics, Hubei Normal University, Huangshi 435002, China \\ Correspondence should be addressed to Tao Xie; 1044806961@qq.com
}

Received 11 March 2021; Accepted 23 June 2021; Published 23 July 2021

Academic Editor: Shiping Wen

Copyright (c) 2021 Wenxiao Si et al. This is an open access article distributed under the Creative Commons Attribution License, which permits unrestricted use, distribution, and reproduction in any medium, provided the original work is properly cited.

\begin{abstract}
With a view to the interference of piecewise constant arguments (PCAs) and neutral terms (NTs) to the original system and the significant applications in the signal transmission process, we explore the robustness of the exponentially global stability (EGS) of recurrent neural network (RNN) with PCAs and NTs (NPRNN). The following challenges arise: what the range of PCAs and the scope of NTs can NPRNN tolerate to be exponentially stable. So we derive two important indicators: maximum interval length of PCAs and the scope of neutral term (NT) compression coefficient here for NPRNN to be exponentially stable. Additionally, we theoretically proved that if the interval length of PCAs and the bound of NT compression coefficient are all lower than the given results herein, the disturbed NPRNN will still remain global exponential stability. Finally, there are two numerical examples to verify the deduced results' effectiveness here.
\end{abstract}

\section{Introduction}

Investigation and synthesis of recurrent neural networks (RNNs) is an unfailing subject regardless of past and present due to its wide application in image and object recognition, speech recognition, model prediction, automatic control, signal processing, and so forth [1-18]. Various stability types for RNNs have been proposed and deeply explored, for instance, asymptotic stability [1-3], exponential stability [4-6], and multistability $[7,8]$. Moreover, some other stability types have been further developed in recent five years, such as synchronization $[9,10]$, impulsive stochastic stability [11], region stability [12], global Mittag-Leffler stabilization [13, 14], memristor-based dynamic behavior stability $[15,16]$, antiperiodic stability [17], exponential Lagrange stability [18] and so on. In fact, compared with the general stability, the study of global exponential stability can be convenient for us to know the convergence rate of the system within a fast time interval accurately and intuitively. Therefore, there is still significative work to be done to explore and characterize global exponential stability or other kinds of stability of recurrent neural networks.
The neutral-type equation is a general class of utility model originating from the mechanical operation, biological neural, economy management, electric circuit, automation control, and the other scientific fields, which have aroused extremely extensive interests from quite a few investigators [19-30]. In terms of electrical implementations, such as mutual reciprocity, package modelling, and the electromagnetic interference design for multiple digital computers, pretty responses had been obtained in certain studies [19]. Therefore, there are several kinds of reasonable featured methods to research the neutral neural network: the one-step method $[20,21]$, block boundary value method [22, 23], Euler-Maclaurin method [24], Runge-Kutta method [25], and Legendre multidomain spectral collocation method [26], while there are very few specific ways to study the robustness of the system by giving the supremum of the neutral term compression coefficient affected by other additional distractions at the same time. It may be a challenge to raise some feasible analytical frameworks to investigate the robustness of recurrent neural networks with NTs (NRNNs). 
Piecewise constant argument (PCA) has a profound influence during the operation process of RNNs. Firstly, the theoretical work on PCA can be traced back to Shah and Joseph [31] and Cooke and Wiener [32]. In quick succession, Akhmet et al. [33] continued to improve this theory and provide evidence to achieve exponentially global stability of RNNs with piecewise constant arguments (PRNNs), appending more integrity with PRNNs at that time. In fact, the method of piecewise argument unifies hysteretic and advance, and can be also applied to other important systems [23, 34, 35], which undoubtedly immensely improved convenience to deal with the impact of time-lag on most systems. Therefore, here we will focus on the discussion of recurrent neural network with piecewise constant arguments and neutral terms (NPRNN) to boost the convenience and completeness of NRNNs.

As far as we know, some of the work is about investigating the robustness of hybrid stochastic models, nonlinear models, and recurrent models equipped with NTs without PCAs [27, 28]. Some of the work has been done to explore the robustness of recurrent models with PCA subjects without NTs [14, 33, 36, 37]. Others have also been deeply done for systems with PCAs and NTs $[23,24,26]$, but they aim to investigate the stability instead of robustness. But there are very few literatures to explore the robustness as well as the globally exponential stability of NPRNNs through giving the interval length of PCAs and the bound of NT compression coefficient together through appropriate parameter settings of PCAs.

Encouraged by these thoughts, this paper will pay attention to the robustness of the globally exponential stability of NPRNN, which can induce faster convergence compared with the general stable system. We will respectively derive the maximum scope of NT compression coefficient and the interval length of PCAs that the system can tolerate to remain stable after interference. First, we may estimate the upper bound of the neutral term compression coefficient $k$ which can be obtained by a linear inequality in one variable. Then, we may select the feasible value of $k$ so that we can successfully determine the supremum of the piecewise argument $\theta$. This has not been fulfilled for the NPRNN model, which is exactly the primary contribution here. Hence, in this paper, the interval length of PCA and the bound of NT compression coefficient for the perturbed NPRNN to be exponentially stable are all provided. Additionally, we will also testify theoretically that if the interval range of the PCAs and the scope of NT compression coefficient of the disturbed system are all lower than the upper bounds given in this paper, then NPRNNs will still remain stable.

The composition of the rest part is set out as follows: the establishment of the NPRNN model, symbol description, and several rational assumptions are presented in Section 2. The upper bounds of the PCA interval length and NT compression coefficient are all provided in Section 3. Finally, two examples and corresponding figures by MATLAB are performed to confirm the main theorems' efficiency in Section 4.

\section{Preliminaries and Notations}

Let $\mathbb{N}, \mathbb{Z}^{+}, \mathbb{R}^{+}$be the natural number set, the positive integer set, and the nonnegative real-number set, respectively. For $n \in \mathbb{N}$, denote $r=\left(r_{1}, r_{2}, \ldots, r_{n}\right)^{T}$ to be a general vector in $\mathbb{R}^{n}$ ( $n$-D real-vector space); the norm of $r$ is expressed as $\|r\|=\sum_{j=1}^{n}\left|r_{j}\right|$, where $T$ is the transpose of a vector. Denote $\underline{N}=\{1,2, \ldots, n\} .\left\{\theta_{q}\right\},\left\{\omega_{q}\right\}$ are two real-valued sequences such that $\theta_{q} \leq \Phi_{q} \leq \theta_{q+1}, \theta_{q} \longrightarrow \infty$ when $k \longrightarrow \infty, q \in \mathbb{N}$.

In the first step, we aim at a kind of RNN model with the effect of neutral terms and generalized piecewise constant arguments (NPRNN), which is written as

$$
\begin{cases}d\left[y_{i}(t)-G_{i}\left(y_{i}(t)\right)\right]=\left[-a_{i} y_{i}(t)+\sum_{j=1}^{n} b_{i j} f_{j}\left(y_{j}(t)\right)+\sum_{j=1}^{n} c_{i j} g_{j}\left(y_{j}(\delta(t))\right)+I_{i}\right] \mathrm{d} t, & t \geq t_{0} \geq 0, \\ y_{i}\left(t_{0}\right)=y_{i}^{0} \in \mathbb{R}, & i \in \underline{N},\end{cases}
$$

where $n$ represents the neurocyte numbers in system (1). $\delta(t)=\varpi_{q}$ for $q \in \mathbb{N}$ and $\theta_{q} \leq t<\theta_{q+1} . G_{i}\left(y_{i}(t)\right): \mathbb{R} \longrightarrow \mathbb{R}$ is the neutral-type function for $y_{i}(t) \in \mathbb{R}$ of the $i$ th neurocyte, where $y_{i}(t)$ is a function which stands for the state trajectory of given time $t$ of the neurocyte $i . f_{j}\left(y_{j}(t)\right)$ and $g_{j}\left(y_{j}(\delta(t))\right)$ represent a class of output (activation functions) at time $t$ and $\delta(t)$ of the jth neurocyte, respectively. $a_{i}$ means the self-inhibition of the $i$ th neurocyte, $a_{i}>0 . b_{i j}$ and $c_{i j}$ are the intensities of the neurocyte $j$ on the neurocyte $i$, corresponding to time $t$ and time $\delta(t)$, respectively. $I_{i}$ refers to the extra interference on the $i$ th neurocyte.
Remark 1. Obviously, system (1) is a class of a hybrid system. That is, if we consider the performance expression of (1) within the interval $\left[\theta_{q}, \theta_{q+1}\right), q \in \mathbb{N}$. System (1) is shown as an advanced system when $\theta_{q} \leq t<\Phi_{q}$, and system (1) is shown as a hysteretic system when $\Phi_{q} \leq t<\theta_{q+1}$. Therefore, system (1) is a mixed system which unifies the advanced and hysteretic systems.

Remark 2. Additionally, compared with (1), in case of $\delta(t)=$ $t$ and without neutral terms, the NPRNN (1) degenerates into the RNN:

$$
\begin{cases}\dot{y}_{i}(t)=-a_{i} y_{i}(t)+\sum_{j=1}^{n} b_{i j} f_{j}\left(y_{j}(t)\right)+\sum_{j=1}^{n} c_{i j} g_{j}\left(y_{j}(t)\right)+I_{i}, & t \geq t_{0} \geq 0, \\ y_{i}\left(t_{0}\right)=y_{i}^{0} \in \mathbb{R}, & i \in \underline{N} .\end{cases}
$$


Apparently, (2) has a zero solution, state $y\left(t, t_{0}, y^{0}\right)$ exists and is unique for any given initial values $t_{0}$ and $y^{0}$ [37]. What follows is the exponentially global stability definition of RNN (2).

Definition 1. Denote that $\check{y}$ is the equilibrium of model (2). State $y(t)$ can achieve exponentially global stability (EGS) if $\|y(t)-\check{y}\|<\bar{\alpha}\|y(t)-\check{y}\| \exp \left\{-\bar{\beta}\left(t-t_{0}\right)\right\}$ exists for all $t \geq t_{0}$, where $\bar{\alpha}, \bar{\beta}>0$. In particular, if $\check{y}=0$ is the equilibrium of model (2), state $y(t)$ can achieve EGS if $\|y(t)\|<\alpha\|y(t)\| \exp \left\{-\beta\left(t-t_{0}\right)\right\}$ is valid, $t \geq t_{0}$, where $\alpha$, $\beta>0$.

To derive the main results, here are some of the assumptions needed:

(A1) $f_{i}, g_{i}$ are all continuous activation functions, $f_{i}, g_{i} \in \mathbb{R}$ for each $i \in \underline{N}$, and $f_{i}(0)=0, g_{i}(0)=0$.

(A2) There are Lipschitz constants $L_{i}^{1}$ and $L_{i}^{2}>0, \zeta_{i}$ and $\eta_{i} \in \mathbb{R}, i \in \underline{N}$, such that

$$
\begin{gathered}
\left|f_{i}\left(\zeta_{i}\right)-f_{i}\left(\eta_{i}\right)\right| \leq L_{i}^{1}\left|\zeta_{i}-\eta_{i}\right|, \\
\left|g_{i}\left(\zeta_{i}\right)-g_{i}\left(\eta_{i}\right)\right| \leq L_{i}^{2}\left|\zeta_{i}-\eta_{i}\right| .
\end{gathered}
$$

(A3) There are Lipschitz constants $k_{i}$, and for any variable $\zeta_{i}, \eta_{i} \in \mathbb{R}, i \in \underline{N}$, we assume

$$
\left|G_{i}\left(\zeta_{i}\right)-G_{i}\left(\eta_{i}\right)\right| \leq k_{i}\left|\zeta_{i}-\eta_{i}\right|, \quad k_{i} \in(0,1) .
$$

Let $k=\max _{i \in N}\left\{k_{i}\right\}, \zeta$ and $\eta \in \mathbb{R}^{n}$; the above inequality can be simply expressed as

$$
\|G(\zeta)-G(\eta)\| \leq k\|\zeta-\eta\|, \quad k \in(0,1) .
$$

(A4) There is a $\theta>0$ such that $\theta_{q+1}-\theta_{q} \leq \theta, q \in \mathbb{N}$.

(A5) $m_{2} \theta+m_{1} \theta\left(m_{2} \theta+1\right) \exp \left\{m_{1} \theta\right\}<1$.

(A6) $\sup _{t \in\left[t_{0}+T, t_{0}+2 T\right]}\|r(t)\| \geq 1$.

(A7) $\alpha \exp (-\beta T)+\left(4 m_{2} T+2 m_{2} T K+k M_{2}\right) \exp \{2 T$ $\left.\left(m_{1}+m_{2}\right)\right\}<1$.

Remark 3. For notational brevity, let

$$
\begin{aligned}
m_{1} & =\max _{1 \leq i \leq n}\left(a_{i}+L_{i}^{1} \sum_{j=1}^{n}\left|b_{j i}\right|\right), \\
m_{2} & =\max _{1 \leq i \leq n}\left(L_{i}^{2} \sum_{j=1}^{n}\left|c_{j i}\right|\right), \\
K & =\frac{K_{1}}{1-\omega_{1}}, \\
K_{1} & =2 k M_{1}\left(1+m_{1} \theta \exp \left\{m_{1} \theta\right\}\right), \\
\rho & =\frac{1}{1-\omega_{1}}, \\
\omega_{1} & =m_{2} \theta+m_{1} \theta\left(1+m_{2} \theta\right) \exp \left\{m_{1} \theta\right\}, \\
M_{1} & =\sup _{t \in\left[\theta_{q}, \theta_{q+1}\right)}\|r(t)\|, \\
v_{3} & =m_{1}+m_{2}, \\
v_{1} & =2 m_{2} T(1+\rho), \\
v_{2} & =2 m_{2} T K, \quad \sup \alpha r(t) \|, \\
& \ln \alpha \\
\left.M_{0}, t_{0}+2 T\right] &
\end{aligned}
$$

where $m_{1}, m_{2}, v_{1}, v_{2}, v_{3}, M_{1}, K_{1}, K, T>0 ; 0<k<1$; and $M_{2} \geq 2$.

\section{Main Results}

In what follows, we aim to give Lemma 1 and Theorem 1 to study the robustness of EGS of the NPRNN system.

At first, we expect to simplify system (1). Actually, we may denote $y_{j}(t)-\check{y}_{j}$ as $r_{j}(t)$ and $y_{j}(\delta(t))-\check{y}_{j}$ as $r_{j}(\delta(t))$. Then, system (1) can be rewritten as a class of NPRNN model (7) as

$$
\begin{cases}d\left[r_{i}(t)-G_{i}\left(r_{i}(t)\right)\right]=\left[-a_{i} r_{i}(t)+\sum_{j=1}^{n} b_{i j} \varphi_{j}\left(r_{j}(t)\right)+\sum_{j=1}^{n} c_{i j} \psi_{j}\left(r_{j}(\delta(t))\right)\right] \mathrm{d} t, & t \geq t_{0} \geq 0, \\ r_{i}\left(t_{0}\right)=r_{i}^{0}=y_{i}^{0} \in \mathbb{R}, & i \in \underline{N},\end{cases}
$$

where $\varphi_{j}\left(r_{j}(t)\right)=f_{j}\left(r_{j}(t)+\check{y}_{j}\right)-f_{j}\left(\check{y}_{j}\right)$ and $\psi_{j}\left(z_{j}\right.$ $(\delta(t)))=g_{j}\left(r_{j}(\delta(t))+\check{y}_{j}\right)-g_{j}\left(\check{y}_{j}\right)$. Generally, we denote $\varphi_{j}(0)=0$ and $\psi_{j}(0)=0, j \in \underline{N}$. In this way, $\varphi_{j}(\cdot)$ and $\psi_{j}(\cdot)$ also satisfy (A2), which are all Lipschitzian with $L_{j}^{1}$ and $L_{j}^{2}$, respectively.

Next, Lemma 1 is given, which directly shows the relationship between the generalized PCA state $r(\delta(t))$ and the state $r(t)$ at present, laying a benign foundation for the proof of the theorem later.

Lemma 1. Define that $r(t)$ is the current state of $(1), r(\delta(t))$ is the state with the generalized PCA of (1), where $t \in \mathbb{R}^{+}$. If assumptions (A1)-(A5) hold, the following inequation exists: 


$$
r(\delta(t)) \leq \rho\|r(t)\|+K
$$

where

$$
\begin{aligned}
\rho & =\left(1-\omega_{1}\right)^{-1}, \\
\omega_{1} & =m_{2} \theta+m_{1} \theta\left(1+m_{2} \theta\right) \exp \left\{m_{1} \theta\right\}, \\
K & =\frac{K_{1}}{1-\omega_{1}}, \\
K_{1} & =2 k M_{1}\left(1+m_{1} \theta \exp \left\{m_{1} \theta\right\}\right), \\
m_{2} & =\max _{1 \leq i \leq n}\left(L_{i}^{2} \sum_{j=1}^{n}\left|c_{j i}\right|\right), \\
m_{1} & =\max _{1 \leq i \leq n}\left(a_{i}+L_{i}^{1} \sum_{j=1}^{n}\left|b_{j i}\right|\right), \\
M_{1} & =\sup _{t \in\left[\theta_{q}, \theta_{q+1}\right.}\|r(t)\| .
\end{aligned}
$$

Proof. For a generalized PCA function $\delta(t)=\varpi_{q}$, define a set $\phi=\left\{t \mid t \in \mathbb{R}^{+}, \theta_{q} \leq t<\theta_{q+1}\right\}$, let $t \in \phi$ and $q \in \mathbb{N}$, and then we have

$$
\begin{aligned}
& r_{i}(t)-r_{i}\left(\oplus_{q}\right)+G_{i}\left(r_{i}\left(\oplus_{q}\right)\right)-G_{i}\left(r_{i}(t)\right) \\
& \leq \int_{\Theta_{q}}^{t}\left[-a_{i} r_{i}(s)+\sum_{j=1}^{n} b_{i j} \varphi_{j}\left(\left(r_{j}(s)\right)\right)+\sum_{j=1}^{n} c_{i j} \psi_{j}\left(r_{j}\left(\oplus_{q}\right)\right)\right] \mathrm{d} s .
\end{aligned}
$$

In combination with (A2), it follows that

$$
\begin{aligned}
& \left\|r(t)-r\left(\Phi_{q}\right)\right\|-\left\|G\left(r\left(\Phi_{q}\right)\right)-G(r(t))\right\| \\
& \leq \sum_{i=1}^{n}\left\{\int_{\Phi_{k}}^{t}\left\{a_{i}\left|r_{i}(s)\right|+\sum_{j=1}^{n} L_{j}^{1}\left|b_{i j}\right|\left|r_{j}(s)\right|+\sum_{j=1}^{n} L_{j}^{2}\left|c_{i j}\right|\left|r_{j}\left(\Phi_{q}\right)\right|\right\} \mathrm{d} s\right\} .
\end{aligned}
$$
that

$$
\begin{aligned}
& \|r(t)\| \leq\left\|G(r(t))-G\left(r\left(\Phi_{q}\right)\right)\right\|+\left\|r\left(\oplus_{q}\right)\right\|+\sum_{i=1}^{n}\left\{\int_{\Phi_{q}}^{t}\left[a_{i}\left|r_{i}(s)\right|+\sum_{j=1}^{n} L_{j}^{1}\left|b_{i j}\right|\left|\left(r_{j}(s)\right)\right|+\sum_{j=1}^{n} L_{j}^{2}\left|c_{i j}\right|\left|r_{j}\left(\Phi_{q}\right)\right|\right] \mathrm{d} s\right\} \\
& \leq\|G(r(t))-G(r(\delta(t)))\|+\left\|r\left(\oplus_{q}\right)\right\|+\left\|G(r(\delta(t)))-G\left(r\left(\oplus_{q}\right)\right)\right\| \\
& \left.+\sum_{i=1}^{n} \int_{\omega_{q}}^{t}\left[a_{i}\left|r_{i}(s)\right|+\sum_{j=1}^{n} L_{j}^{1}\left|b_{i j}\right|\left(r_{j}(s)\right)\left|+\sum_{j=1}^{n} L_{j}^{2}\right| c_{i j}|| r_{j}\left(\oplus_{q}\right) \mid\right] \mathrm{d} s\right\} \\
& \leq k\left\|r(t)-r\left(\Phi_{q}\right)\right\|+\left\|r\left(\varpi_{q}\right)\right\|+\sum_{i=1}^{n}\left\{\int_{\Phi_{q}}^{t}\left[a_{i}\left|r_{i}(s)\right|+\sum_{j=1}^{n} L_{j}^{1}\left|b_{i j}\right|\left|\left(r_{j}(s)\right)\right|+\sum_{j=1}^{n} L_{j}^{2}\left|c_{i j}\right|\left|r_{j}\left(\Phi_{q}\right)\right|\right] \mathrm{d} s\right\} \\
& \leq k\left\|r(t)-r\left(\Phi_{q}\right)\right\|+\left\|r\left(\oplus_{q}\right)\right\|+\int_{\Theta_{q}}^{t}\left[\sum_{i=1}^{n}\left(a_{i}\left|r_{i}(s)\right|+L_{i}^{1} \sum_{j=1}^{n}\left|b_{j i}\right|\left|r_{i}(s)\right|+L_{i}^{2} \sum_{j=1}^{n}\left|c_{j i}\right|\left|r_{i}\left(\Phi_{q}\right)\right|\right)\right] \mathrm{d} s \\
& \leq 2 k \sup _{t \in\left[\theta_{q}, \theta_{q+1}\right)}\|r(t)\|+\left\|r\left(\Theta_{q}\right)\right\|+\int_{\Theta_{q}}^{t}\left[\sum_{i=1}^{n}\left(a_{i}\left|r_{i}(s)\right|+L_{i}^{1} \sum_{j=1}^{n}\left|b_{j i}\right|\left|r_{i}(s)\right|+L_{i}^{2} \sum_{j=1}^{n}\left|c_{j i}\right|\left|r_{i}\left(\Theta_{q}\right)\right|\right)\right] \mathrm{d} s \\
& \leq 2 k M_{1}+\left\|r\left(\Phi_{q}\right)\right\|+\int_{\omega_{q}}^{t}\left[\sum_{i=1}^{n}\left(a_{i}+L_{i}^{1} \sum_{j=1}^{n}\left|b_{j i}\right|\right)\left|r_{i}(s)\right|+\sum_{i=1}^{n} \sum_{j=1}^{n} L_{i}^{2}\left|c_{j i}\right|\left|r_{i}\left(\Phi_{q}\right)\right|\right] \mathrm{d} s \\
& \leq 2 k M_{1}+\left\{1+\int_{\omega_{q}}^{t} \sum_{i=1}^{n}\left(L_{i}^{2} \sum_{j=1}^{n}\left|c_{j i}\right|\right)\left|r_{i}\left(\Theta_{q}\right)\right| \mathrm{d} s\right\}+\int_{\omega_{q}}^{t}\left[\sum_{i=1}^{n}\left(a_{i}+L_{i}^{1} \sum_{j=1}^{n}\left|b_{j i}\right|\right)\left|r_{i}(s)\right|\right] \mathrm{d} s \\
& \leq 2 k M_{1}+\left\{1+\max _{1 \leq i \leq n}\left\{L_{i}^{2} \sum_{j=1}^{n}\left|c_{j i}\right|\right\} \int_{\omega_{q}}^{t}\left(\sum_{i=1}^{n}\left|r_{i}\left(\varpi_{q}\right)\right|\right) \mathrm{d} s\right\}+\max _{1 \leq i \leq n}\left\{a_{i}+L_{i}^{1} \sum_{j=1}^{n}\left|b_{j i}\right|\right\} \\
& \times \int_{\omega_{\mathcal{q}}}^{t}\left(\sum_{i=1}^{n}\left|r_{i}(s)\right|\right) \mathrm{d} s \\
& \leq 2 k M_{1}+\left(1+m_{2} \theta\right)\left\|r\left(\Theta_{q}\right)\right\|+\int_{\omega_{q}}^{t} m_{1}\|r(s)\| \mathrm{d} s,
\end{aligned}
$$


where $M_{1}=\sup _{t \in\left[\theta_{q}, \theta_{q+1}\right.}\|r(t)\|$. Besides, $m_{1}$ and $m_{2}$ are defined in Remark 3. derive

$$
\|r(t)\| \leq\left[\left(1+m_{2} \theta\right)\left\|r\left(\bigotimes_{q}\right)\right\|+2 k M_{1}\right] \exp \left\{m_{1} \theta\right\} .
$$

Otherwise, for $\theta_{q} \leq t<\theta_{q+1}$, similarly, we get

$$
\begin{aligned}
& \left\|r\left(\varpi_{q}\right)\right\| \leq 2 k M_{1}+\|r(t)\|+\int_{\Phi_{q}}^{t}\left[\sum_{i=1}^{n}\left(a_{i}+L_{i}^{1}\left|b_{j i}\right|\right)\left|r_{i}(s)\right|+\sum_{i=1}^{n} \sum_{j=1}^{n} L_{i}^{2}\left|c_{j i}\right|\left|r_{i}\left(\varpi_{q}\right)\right|\right] \mathrm{d} s \\
& \leq 2 k M_{1}+\|r(t)\|+m_{2} \theta\left\|r\left(\oplus_{q}\right)\right\|+\int_{\omega_{q}}^{t} m_{1}\|r(s)\| \mathrm{d} s \\
& \leq 2 k M_{1}+\|r(t)\|+m_{2} \theta\left\|r\left(\Phi_{q}\right)\right\|+m_{1} \theta \exp \left\{m_{1} \theta\right\}\left[\left(1+m_{2} \theta\right)\left\|r\left(\varpi_{q}\right)\right\|+2 k M_{1}\right] \\
& \leq\|r(t)\|+\left[m_{2} \theta+m_{1} \theta \exp \left\{m_{1} \theta\right\}\left(1+m_{2} \theta\right)\right]\left\|r\left(\bigotimes_{q}\right)\right\|+2 k M_{1}\left(1+m_{1} \theta \exp \left\{m_{1} \theta\right\}\right) \\
& \leq\|r(t)\|+\omega_{1}\left\|r\left(\varpi_{q}\right)\right\|+K_{1},
\end{aligned}
$$

where $\omega_{1}=m_{2} \theta+m_{1} \theta\left(1+m_{2} \theta\right) \exp \left\{m_{1} \theta\right\}, K_{1}=2 k M_{1}(1+$ $\left.m_{1} \theta \exp \left\{m_{1} \theta\right\}\right)$ and $m_{1}, m_{2}$, and $M_{1}$ are defined in Remark 3.

Consequently, by combining the above equation with similar terms, we can obtain

$$
\left(1-\omega_{1}\right)\left\|r\left(\varpi_{q}\right)\right\| \leq\|r(t)\|+K_{1} .
$$

So, for $\delta(t)=\varpi_{q}$ and $\omega_{1}<1$ for (A5), we can get

$$
\begin{aligned}
& \|r(\delta(t))\| \leq\left\{1-\omega_{1}\right\}^{-1} \cdot\|r(t)\|+\left\{1-\omega_{1}\right\}^{-1} \cdot K_{1} \\
& \quad=: \rho\|r(t)\|+K,
\end{aligned}
$$

where $\rho=1 /\left(1-\omega_{1}\right)$ and $K=K_{1} /\left(1-\omega_{1}\right)$. In this way, (8) is valid for any $t \geq 0$. And the proof is over.

Remark 4. Different from the previous recurrent neural network with piecewise arguments [37], Lemma 1 has one more term than the previous lemma in [37] due to the presence of neutral terms. It is reasonable, which shows the impact of neutral terms on PRNN.

Theorem 1. If (A1)-(A7) exist and RNN (2) achieves exponentially global stability. And if

$$
\begin{aligned}
& k<\left(2 M_{1}+M_{2}\right)^{-1}\left\{[1-\alpha \exp (-\beta T)] \exp \left\{-2\left(m_{1}+m_{2}\right) T\right\}-4 m_{2} T\right\}, \\
& \theta<\theta_{4}=\min \left\{\theta_{3}, \min \left\{\sup \left\{\theta_{1}, \theta_{2}\right\}\right\}\right\}
\end{aligned}
$$

where $\theta_{3}>0, \theta_{3}$ is written as the only solution to the following transcendental equation:

$$
\alpha \exp (-\beta T)+\left(\nu_{1}+\nu_{2}+k M_{2}\right) \exp \left\{2 v_{3} T\right\}=1 .
$$

$\theta_{1}$ and $\theta_{2}$ satisfy (A5) and (A7), respectively; then, NPRNN (7) can be globally exponentially stable, where

$$
\begin{aligned}
v_{1} & =2 m_{2} T(1+\rho), \\
v_{2} & =2 m_{2} T K, \\
v_{3} & =m_{1}+m_{2}, \\
M_{2} & =2 \sup _{t_{0} \leq t \leq t_{0}+2 T}\|r(t)\| .
\end{aligned}
$$

Besides, $m_{1}, m_{2}, T, M_{1}, \rho, \omega_{1}, K$, and $K_{1}$ here are the same as those in Lemma 1.

Proof. For convenience, denote $y(t)$ to be shorthand for $y\left(t, t_{0}, y^{0}\right)$ and $r(t)$ to be shorthand for $r\left(t, t_{0}, r^{0}\right)$, which are the states of systems (2) and (7), respectively. Thus from (2) and (7), we have

$$
\begin{aligned}
& y_{i}(t)-r_{i}(t)+G_{i}\left(r_{i}(t)\right)-G_{i}\left(r_{i}^{0}\right) \\
& =\int_{t_{0}}^{t}\left\{-a_{i}\left(y_{i}(s)-r_{i}(s)\right)+\sum_{j=1}^{n} b_{i j}\left(f_{j}\left(y_{j}(s)\right)-f_{j}\left(r_{j}(s)\right)\right)+\sum_{j=1}^{n} c_{i j}\left(g_{j}\left(y_{j}(s)\right)-g_{j}\left(r_{j}(\delta(s))\right)\right)\right\} \mathrm{d} s .
\end{aligned}
$$


Utilize (A2) and norm inequation for (20), $i \in \underline{N}$; that is

$$
\begin{aligned}
\| y( & t)-r(t)\|-\| G(r(t))-G\left(r^{0}\right) \| \\
\leq & \sum_{i=1}^{n}\left\{\int _ { t _ { 0 } } ^ { t } \left[a_{i}\left|y_{i}(s)-r_{i}(s)\right|+\sum_{j=1}^{n} L_{j}^{1}\left|b_{i j}\right|\left|y_{j}(s)-r_{j}(s)\right|\right.\right. \\
& \left.\left.+\sum_{j=1}^{n} L_{j}^{2}\left|c_{i j}\right|\left|y_{j}(s)-r_{j}(\delta(s))\right|\right] \mathrm{d} s\right\} \\
\leq & \sum_{i=1}^{n}\left\{\int _ { t _ { 0 } } ^ { t } \left[a_{i}\left|y_{i}(s)-r_{i}(s)\right|+\sum_{j=1}^{n} L_{j}^{1}\left|b_{i j}\right|\left|y_{j}(s)-r_{j}(s)\right|\right.\right. \\
& \left.\left.\left.+\sum_{j=1}^{n} L_{j}^{2}\left|c_{i j}\right|\left(\left|y_{j}(s)-r_{j}(s)\right|+\left|r_{j}(s)-r_{j}(\delta(s))\right|\right)\right] \mathrm{d}\right\}\right\} \\
\leq & \int_{t_{0}}^{t}\left[\sum_{i=1}^{n}\left|a_{i}\right|\left|y_{i}(s)-r_{i}(s)\right|+\sum_{i=1}^{n}\left(L_{i}^{1} \sum_{j=1}^{n}\left|b_{j i}\right|+L_{i}^{2} \sum_{j=1}^{n}\left|c_{j i}\right|\right)\left|y_{i}(s)-r_{i}(s)\right|\right. \\
& +\sum_{i=1}^{n} \sum_{j=1}^{n} L_{i}^{2}\left|c_{j i}\right|\left|r_{i}(s)-r_{i}(\delta(s))\right| \mathrm{d} s .
\end{aligned}
$$

Based on Lemma 1 and Remark 3, for $t_{0} \leq t \leq t_{0}+2 T$, we can derive

$$
\begin{aligned}
& \|y(t)-r(t)\| \\
& \leq \int_{t_{0}}^{t} \max _{i \in \underline{N}}\left\{\left|a_{i}\right|+L_{i}^{1} \sum_{j=1}^{n}\left|b_{j i}\right|+L_{i}^{2} \sum_{j=1}^{n}\left|c_{j i}\right|\right\}\|y(s)-r(s)\| \mathrm{d} s \\
& \quad+\int_{t_{0}}^{t} \max _{i \in \underline{N}}\left\{L_{i}^{2} \sum_{j=1}^{n}\left|c_{j i}\right|\right\}\|r(s)-r(\delta(s))\| \mathrm{d} s+\left\|G(r(t))-G\left(r^{0}\right)\right\| \\
& \leq\left(m_{1}+m_{2}\right) \int_{t_{0}}^{t}\|y(s)-r(s)\| \mathrm{d} s+m_{2} \int_{t_{0}}^{t}(\|r(\delta(s))\|+\|r(s)\|) \mathrm{d} s+2 k \sup _{t_{0} \leq t \leq t_{0}+2 T}\{\|r(t)\|\} \\
& \leq\left(m_{1}+m_{2}\right) \int_{t_{0}}^{t}\|y(s)-r(s)\| \mathrm{d} s+2 m_{2} T(1+\rho)\|(r(t))\|+2 m_{2} T K+k M_{2} \\
& \leq v_{1}\|r(t)\|+k M_{2}+v_{2}+v_{3} \int_{t_{0}}^{t}\|y(s)-r(s)\| \mathrm{d} s,
\end{aligned}
$$

where

$$
\begin{aligned}
v_{1} & =2 m_{2} T(1+\rho), \\
v_{2} & =2 m_{2} T K, \\
v_{3} & =m_{1}+m_{2}, \\
M_{2} & =2 \sup _{t_{0} \leq t \leq t_{0}+2 T}\|r(t)\| .
\end{aligned}
$$


Applying the Gronwall inequation and (A6), for $t_{0}+T \leq t \leq t_{0}+2 T$, we derive\&ecmath;

$$
\begin{aligned}
\|y(t)-r(t)\| & \leq\left(v_{2}+k M_{2}+v_{1}\|r(t)\|\right) \exp \left\{2 v_{3} T\right\} \\
& \leq\left(v_{2}+k M_{2}\right) \sup _{t \in\left[t_{0}+T, t_{0}+2 T\right]}\{\|r(t)\|\} \cdot \exp \left\{2 v_{3} T\right\}+v_{1} \sup _{t \in\left[t_{0}+T, t_{0}+2 T\right]}\{\|r(t)\|\} \cdot \exp \left\{2 v_{3} T\right\} \\
& \leq\left(v_{1}+v_{2}+k M_{2}\right) \exp \left\{2 v_{3} T\right\} \sup _{t \in\left[t_{0}+T, t_{0}+2 T\right]}\{\|r(t)\|\},
\end{aligned}
$$

for $\left(\nu_{2}+k M_{2}\right) \exp \left\{2 \nu_{3} T\right\}>0$ in Remark 3 .

Subsequently, by Definition 1 and (24), for $t_{0}+T \leq t \leq t_{0}+2 T$, we further have

$$
\begin{aligned}
\|r(t)\| & \leq\|y(t)-r(t)\|+\|y(t)\| \\
& \leq\left(v_{1}+v_{2}+k M_{2}\right) \exp \left\{2 v_{3} T\right\} \sup _{t \in\left[t_{0}+T, t_{0}+2 T\right]}\{\|r(t)\|\}+\|y(t)\| \\
& \leq\left[\left(v_{1}+v_{2}+k M_{2}\right) \exp \left\{2 v_{3} T\right\}+\alpha \exp \left(-\beta\left(t-t_{0}\right)\right)\right] \sup _{t \in\left[t_{0}+T, t_{0}+2 T\right]}\{\|r(t)\|\} \\
& \leq\left[\left(v_{1}+v_{2}+k M_{2}\right) \exp \left\{2 v_{3} T\right\}+\alpha \exp (-\beta T)\right] \sup _{t \in\left[t_{0}+T, t_{0}+2 T\right]}\{\|r(t)\|\} \\
& =\widehat{C} \sup _{t \in\left[t_{0}+T, t_{0}+2 T\right]}\{\|r(t)\|\},
\end{aligned}
$$

with $\widehat{C}=\alpha \exp (-\beta T)+\left(\nu_{1}+\nu_{2}+k M_{2}\right) \exp \left\{2 \nu_{3} T\right\}$.

Denote

$$
\begin{aligned}
F(\rho, k) & =\alpha \exp (-\beta T)+\left(\nu_{1}+v_{2}+k M_{2}\right) \exp \left\{2 v_{3} T\right\} \\
& =\alpha \exp (-\beta T)+\left[2 m_{2} T(1+\rho)+2 m_{2} T K+k M_{2}\right] \exp \left\{2\left(m_{1}+m_{2}\right) T\right\}
\end{aligned}
$$

since $F(\rho, k)$ is strictly and continuously monotonous in terms of variable $\rho$ on the interval $[1,+\infty)$, from Theorem 1 and (A7), we have

$$
F(1, k)=\alpha \exp (-\beta T)+\left(4 m_{2} T+2 m_{2} T K+k M_{2}\right) \exp \left\{2\left(m_{1}+m_{2}\right) T\right\}<1,
$$

and

$$
F(\infty, k)>1 \text {. }
$$

Hence, on the one hand, there is a unique $\tilde{\rho} \in(1,+\infty)$ which makes $F(\widetilde{\rho}, k)=1$ true. From assumption (A5), let

$$
\Gamma(\theta)=m_{2} \theta+m_{1} \theta\left(1+m_{2} \theta\right) \exp \left\{m_{1} \theta\right\}<1,
$$

such that $\theta_{1}\left(\theta_{1}>0\right)$ satisfy inequation (29), and surely $\theta_{1}$ satisfy (A5). Obviously,

$$
\rho(\theta)=\frac{1}{1-\Gamma(\theta)} \in(1,+\infty)
$$

holds towards any $\theta \in\left(0, \theta_{1}\right)$. Hence, there is a unique $\widetilde{\theta} \in\left[0, \theta_{1}\right)$, which makes it possible for $\rho=\tilde{\rho}$. Then, if we assume that $\theta_{2}$ satisfies (27), $\theta_{3}$ is the unique positive solution of (26).

Let

$$
\theta_{4}=\min \left\{\theta_{3}, \min \left\{\sup \left\{\theta_{1}, \theta_{2}\right\}\right\},\right.
$$

by virtue of the monotonicity of $F(\cdot)$ and $\rho(\cdot)$, we know that $0<F(\rho, k)=\widehat{C}<1$ holds when $0<\theta<\theta_{4}$, and we obtain the interval length of PCA so far.

On the other hand, surely, we know that there is some $\widetilde{k}<k$ such that $F(1, \widetilde{k})<1$ from (27). Let $\theta=0$ in (27); then the bound to $k$ can be described as 
$k<\left(2 M_{1}+M_{2}\right)^{-1}\left\{[1-\alpha \exp (-\beta T)] \exp \left\{-2\left(m_{1}+m_{2}\right) T\right\}-4 m_{2} T\right\}$,

Denote $\xi=-(\ln \widehat{C}) / T$; according to $(25)$, we can obtain

$$
\sup _{t_{0}+T \leq t \leq t_{0}+2 T}\|r(t)\| \leq \exp (-\xi T) \sup _{t_{0} \leq t \leq t_{0}+T}\|r(t)\| .
$$

Consequently, for any positive integer $l \in \mathbb{Z}^{+}$, when $t \geq t_{0}+(l-1) T$, by the uniqueness of solution [27], we can express the solutions of the NPRNN in the following flow modality:

$$
r(t) \equiv r\left(t ; t_{0}, r^{0}\right)=r\left(t ; t_{0}+(l-1) T, r\left(t_{0}+(l-1) T ; t_{0}, r^{0}\right)\right) .
$$

Taking (33) and (34) into account, it follows that

$$
\begin{aligned}
& \sup _{t_{0}+l T \leq t \leq t_{0}+(l+1) T}\|r(t)\| \\
& =\sup _{t_{0}+(l-1) T+T \leq t \leq t_{0}+(l-1) T+2 T}\left\|r\left(t ; t_{0}+(l-1) T, r\left(t_{0}+(l-1) T ; t_{0}, r^{0}\right)\right)\right\| \\
& \leq \exp (-\xi T) \sup _{t_{0}+(l-1) T \leq t \leq t_{0}+l T}\|r(t)\| \\
& \leq \exp (-l \xi T) \sup _{t \in\left[t_{0}, t_{0}+T\right]}\|r(t)\| \\
& =C \exp (-l \xi T)
\end{aligned}
$$

where $C=\sup _{t \in\left[t_{0}, t_{0}+T\right]}\|r(t)\|$. Hence, in the time interval $\left[t_{0}+l T, t_{0}+(l+1) T\right], l \in \mathbb{Z}^{+}$, we obtain

$$
\begin{aligned}
\|z(t)\| & \leq C \exp (-l \xi T) \\
& \leq C \exp \left(\xi\left(-t+t_{0}+T\right)\right) \\
& \leq C \exp (\xi T) \exp \left(-\xi\left(t-t_{0}\right)\right) .
\end{aligned}
$$

In this way, the NPRNN model (7) achieves exponentially global stability.

Remark 5. Few studies have been finished to comprehensively consider the influence of robustness and exponentially global stability on NPRNN model by giving the upper bounds of PCAs and NTs. In this paper, from Lemma 1, by the method of norm scaling inequality technique, the relationship between the generalized PCA state $r(\delta(t))$ and the state $r(t)$ at present is clarified. From Theorem 1, by utilizing Gronwall Lemma, norm scaling inequality technique, and the scientific method of controlling variables, the interval length of PCAs and the maximum scope of NT compression coefficient of NPRNN (7) are derived intuitively.
Remark 6. The interval length of PCAs and the maximum scope of NT compression coefficient can be estimated by software tool MATLAB. The order of calculation is as follows: firstly, we choose suitable $T=(\ln \alpha / \beta)>0$. Furthermore, since $\alpha, \beta, L_{i}^{1}$ and $L_{i}^{2}$ are known and other parameters can be computed in terms of Remark 3, $i \in \underline{N}$. Thus, the bound of $k$ can be calculated easily by (32). Additionally, we select the appropriate $k$ and substitute it into transcendental equation (18) with other parameters together so that the length of the argument interval can be acquired. Finally, a comprehensive selection of values that satisfy both $k$ and $\theta$ inequality conditions in Theorem 1 can ensure the exponential stability of NPRNN (7).

\section{Numerical Examples}

For the sake of exploring the robustness of the EGS of NPRNN, we derive two important indicators: the supremum of the interval length of PCA $\theta$ and the feasible upper range of NT compression coefficient $k$ in this paper. Thus, there are two elucidative examples to testify the efficiency for the criteria in Theorem 1.

Example 1. The following bistate RNN is considered:

$$
\left(\begin{array}{c}
\dot{y}_{1}(t) \\
\dot{y}_{2}(t)
\end{array}\right)=\left(\begin{array}{cc}
-2 & 0 \\
0 & -1
\end{array}\right)\left(\begin{array}{l}
y_{1}(t) \\
y_{2}(t)
\end{array}\right)+\left(\begin{array}{cc}
0.02 & 0.01 \\
0.01 & 0.02
\end{array}\right)\left(\begin{array}{c}
\sin \left(\frac{y_{1}(t)}{20}\right) \\
\sin \left(\frac{y_{2}(t)}{20}\right)
\end{array}\right)
$$


By calculation, we easily get that RNN (37) can achieve exponentially global stability when we set suitable parameters $\alpha=1, \beta=0.5$; one can see Figures 1 and 2 .
Next, in the presence of the neutral terms and piecewise constant arguments, (37) becomes

$$
\begin{aligned}
\frac{\mathrm{d}}{\mathrm{d} t}\left(\begin{array}{c}
r_{1}(t)-k \sin \left(r_{1}(t)\right) \\
r_{2}(t)-k \sin \left(r_{2}(t)\right)
\end{array}\right)= & \left(\begin{array}{cc}
-2 & 0 \\
0 & -1
\end{array}\right)\left(\begin{array}{c}
r_{1}(t) \\
r_{2}(t)
\end{array}\right)+\left(\begin{array}{cc}
0.02 & 0.01 \\
0.01 & 0.02
\end{array}\right)\left(\begin{array}{c}
\sin \left(\frac{r_{1}(t)}{20}\right) \\
\sin \left(\frac{r_{2}(t)}{20}\right)
\end{array}\right) \\
& +\left(\begin{array}{cc}
-0.001 & 0.002 \\
0.002 & -0.001
\end{array}\right)\left(\begin{array}{c}
\sin \left(r_{1}(\delta(t))\right) \\
\sin \left(r_{2}(\delta(t))\right)
\end{array}\right) .
\end{aligned}
$$

Fix two consequences: $\left\{\theta_{q}\right\}=\{q / 9\},\left\{\varpi_{q}\right\}=\{(2 q+1)$ $/ 18\}, q \in \mathbb{N}$. Define activation functions $f_{j}\left(r_{j}(t)\right)$ and $g_{j}\left(r_{j}(t)\right)$ here as $\sin \left(r_{j}(t) / 20\right)$ and $\sin \left(r_{j}(\delta(t))\right)$, respectively, so we have $L_{1}^{1}=L_{2}^{1}=1 / 20, L_{1}^{2}=L_{2}^{2}=1$, where $j=1,2$. Then by calculation, we can obtain $m_{1}=2.0015$, $m_{2}=0.001, v_{3}=2.0025$.

According to Theorem 1 and (32), let $T=0.3>((\ln \alpha)$ $/ \beta)=0$, and then we have

$$
k<0.25 \times\{[1-\exp (-0.15)] \times \exp (-1.2015)-0.0012\}=0.0102 .
$$

From Theorem 1, if we set

$$
f_{1}(\theta)=m_{2} \theta+m_{1} \theta\left(1+m_{2} \theta\right) \exp \left\{m_{1} \theta\right\}=1,
$$

the unique positive solution of (40) $\tilde{\theta}_{1}=0.2833$ is the supremum of (A5). Solving

$$
\begin{aligned}
f_{2}(\theta)= & \alpha \exp (-\beta T) \\
& +\left(4 m_{2} T+2 m_{2} T K+k M_{2}\right) \exp \left\{2\left(m_{1}+m_{2}\right) T\right\}=1,
\end{aligned}
$$

the unique positive solution of (41) $\tilde{\theta}_{2}=0.2832$ is the supremum of (A7). So we get

$$
\min \left\{\tilde{\theta_{1}}, \tilde{\theta_{2}}\right\}=0.2832 \text {. }
$$

Let $\theta_{3}\left(\theta_{3}>0\right)$ be the supremum of equation (18); then we can obtain $\theta_{3}=0.2829$ by MATLAB. So

$$
\theta<\theta_{4}=\min \left\{\theta_{3}, \min \left\{\tilde{\theta}_{1}, \tilde{\theta}_{2}\right\}\right\}=\min \{0.2829,0.2832\}=0.2829 \text {. }
$$

Due to the monotonicity of $f_{1}(\theta)$ and $f_{2}(\theta)$ in (40) and (41), it is easy to get that (A5) and (A7) hold when $\theta=1 / 9$, $k=0.01$.

So, in the case that if the real selected values are all lower than the derived values, that is, the criteria in Theorem 1 and (A1)-(A7) are all satisfied, NPRNN (7) will be exponentially globally stable if we take $\theta=1 / 9=0.1111<\theta_{4}=0.2829$ and $k=0.01<0.0102$. Then the simulations of the NPRNN model with the interval length of the argument $\theta=1 / 9$ and the bound to neutral term compression coefficient $k=0.01$ of state $r_{1}(t)$ and $r_{2}(t)$ are given in Figures 3 and 4 . That is to say, if the interval length of PCA $\theta$ and the neutral term compression coefficient $k$ are all lower than the results given in Theorem 1, transient behavior will remain exponentially stable.

Example 2. The following one-dimensional dynamical system is considered:

$$
\dot{y}(t)=-3.1 y(t)+0.1 f(y(t)) .
$$

Let $f(\cdot)=\tanh (\cdot)$, according to many existing criteria, the neurodynamic system (44) can be stable when we take $\alpha=1, \beta=3$. Figure 5 illustrates the convergence stability of RNN (44).

In what follows, if we add neutral terms and piecewise constant arguments to system (44), we can get the NPRNN (45) as

$$
\frac{\mathrm{d}}{\mathrm{d} t}(r(t)-k \sin r(t))=-3.1 r(t)+0.088 \tanh (r(t))+0.002 \tan h(r(\delta(t)))
$$

Similarly, if we fix two consequences $\left\{\theta_{q}\right\}=\{q / 10\}$ and $\left\{\omega_{q}\right\}=\{(2 q+1) / 20\}$, according to the characteristics of (45), we can easily get $L_{1}^{1}=L_{1}^{2}=L_{2}^{1}=L_{2}^{2}=1$. Then the parameters that we need by easy calculation are listed as $m_{1}=3.188$, 


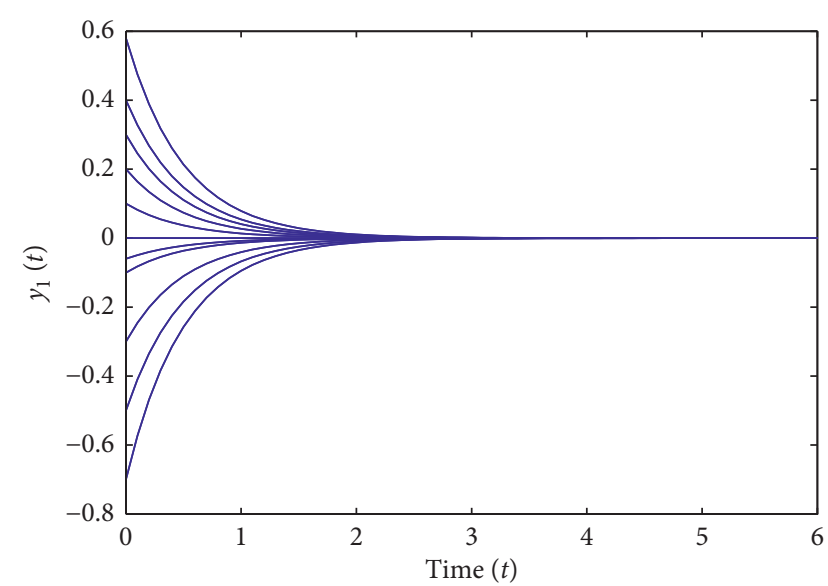

Figure 1: Stable behavior of $y_{1}(t)$ of RNN (37).

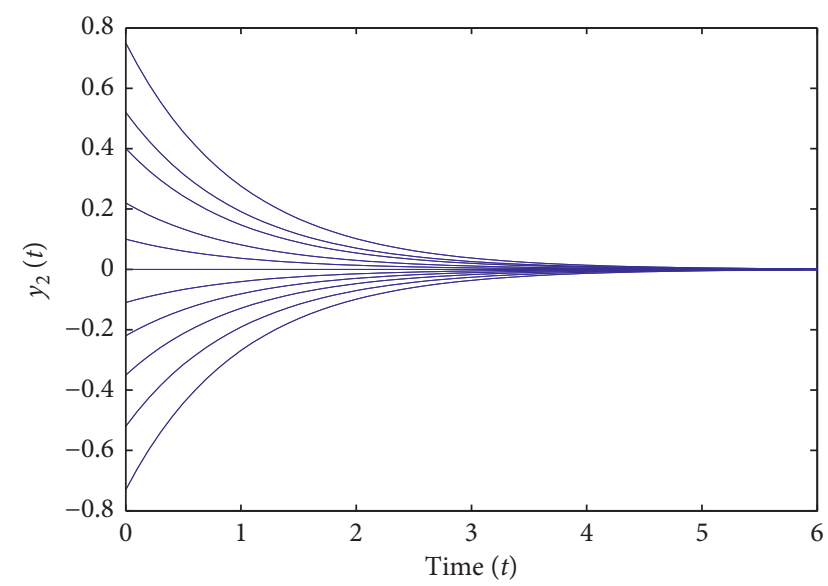

FIgURE 2: Stable behavior of $y_{2}(t)$ of RNN (37).

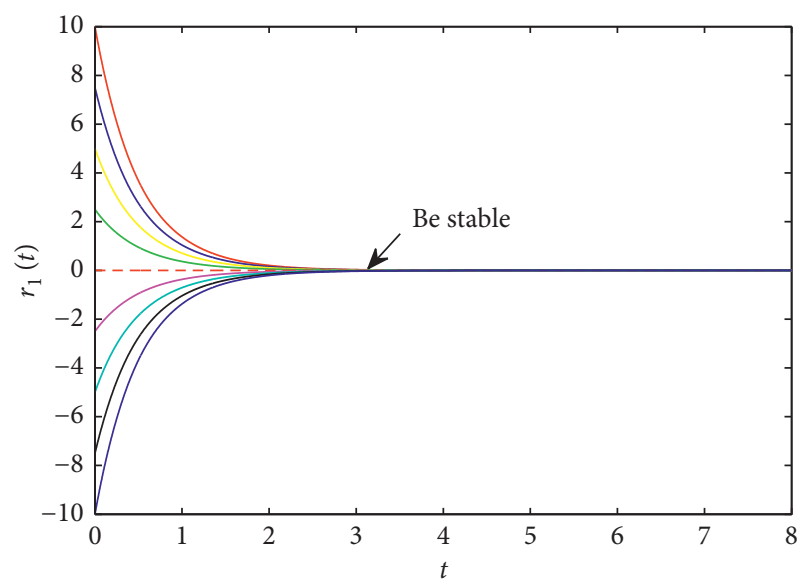

Figure 3: The EGS of $r_{1}(t)$ with $\theta=(1 / 9), k=0.01$ in (38).

$m_{2}=0.002, v_{3}=3.19$. By Theorem 1 , let $T=0.25>((\ln \alpha)$ $/ \beta)=0$. Substituting them into (32), we get

$k<0.25 \times\{[1-\exp (-0.75)] \times \exp (-1.595)-0.002\}=0.0263$.

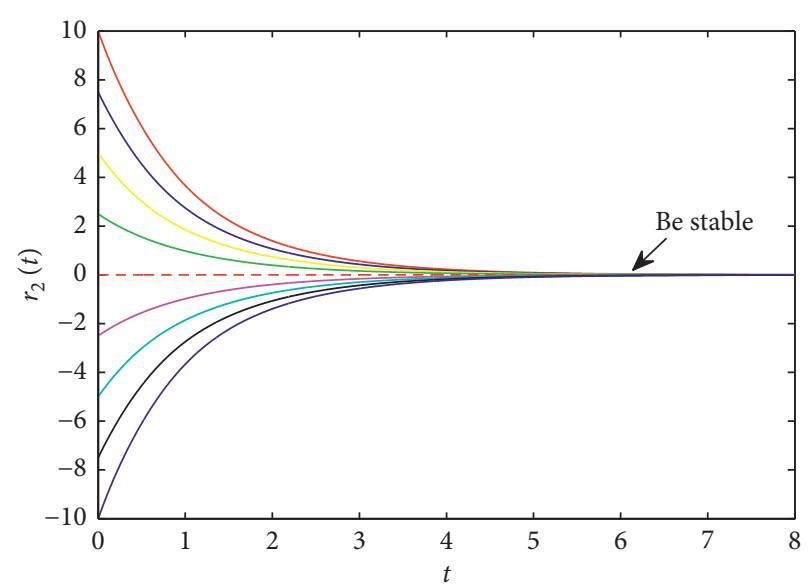

Figure 4: The EGS of $r_{2}(t)$ with $\theta=(1 / 9), k=0.01$ in (38).

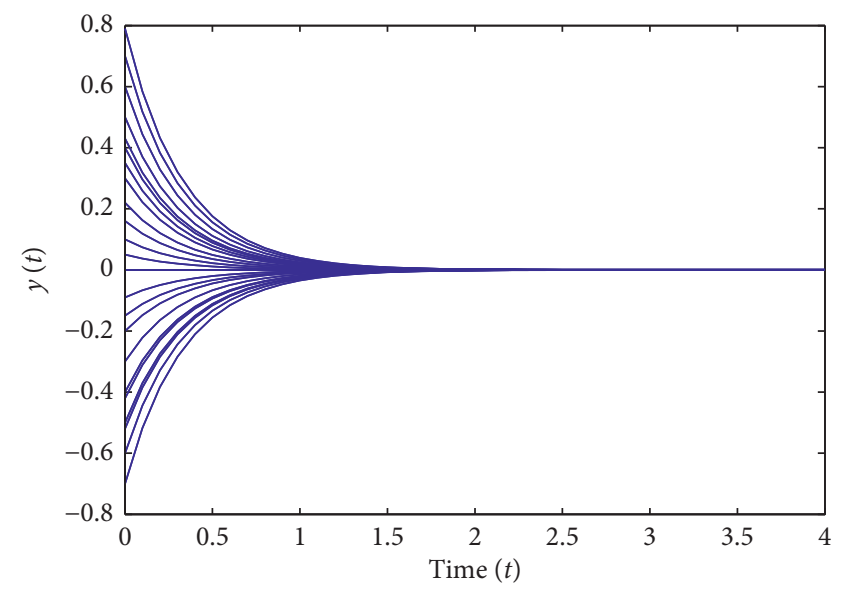

Figure 5: Stable trajectory of $y(t)$ in (44).

Suppose that $\theta_{1}$ and $\theta_{2}$ satisfy $(A 5)$ and $(A 7)$, respectively. Put the parameters of Remark 3 into $(A 5)$ and $(A 7)$, respectively, and we can get

$$
\min \left\{\sup \left\{\theta_{1}, \theta_{2}\right\}\right\}=0.1778 \text {. }
$$

Next, we can get $\theta_{3}=0.1774$ in Theorem 1 by using MATLAB when other essential parameters are provided; then we obtain that

$$
\theta_{4}=\min \left\{\theta_{3}, \min \left\{\sup \left\{\theta_{1}, \theta_{2}\right\}\right\}\right\}=0.1774 \text {. }
$$

Moreover, since the increasing monotonicity of exponential function (26), the solution $\theta_{4}=0.1774$ also satisfies assumptions $(A 5)$ and $(A 7)$, respectively. Hence, the conditions in the assumptions and theorem are all satisfied. So if we take $k=0.01, \theta=0.1$, the convergence stability of $r(t)$ of NPRNN system (45) can be seen in Figure 6.

Furthermore, several unstable cases are given to illustrate.

In Figure 7 , where $\theta=0.1, k=1.02$, which are not suitable for conditions in Theorem 1 , the state trajectories of NPRNN (45) are unstable.

In Figure 8, where $k=0.01, \theta=0.5$, which are not fit for the conditions in Theorem 1, the state trajectories of NPRNN (45) are unstable. 


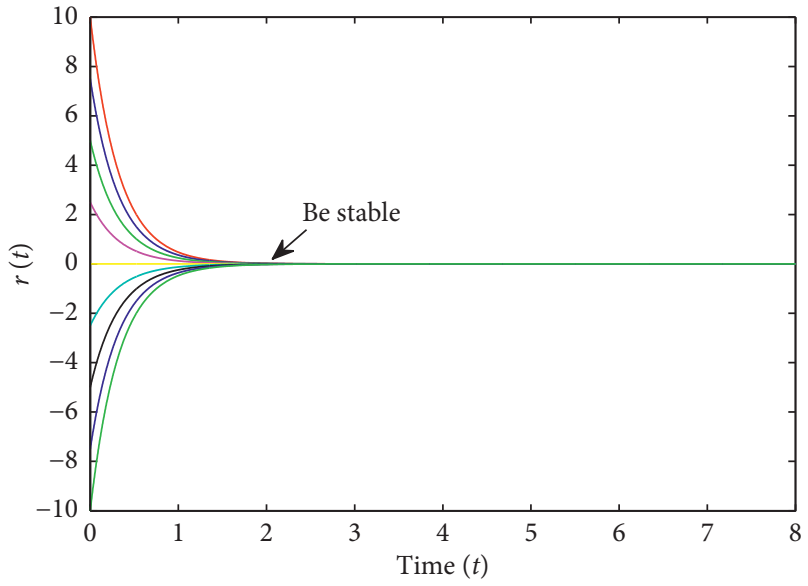

FIGURE 6: The EGS of $r(t)$ with $k=0.01$ and $\theta=0.1$ in (45).

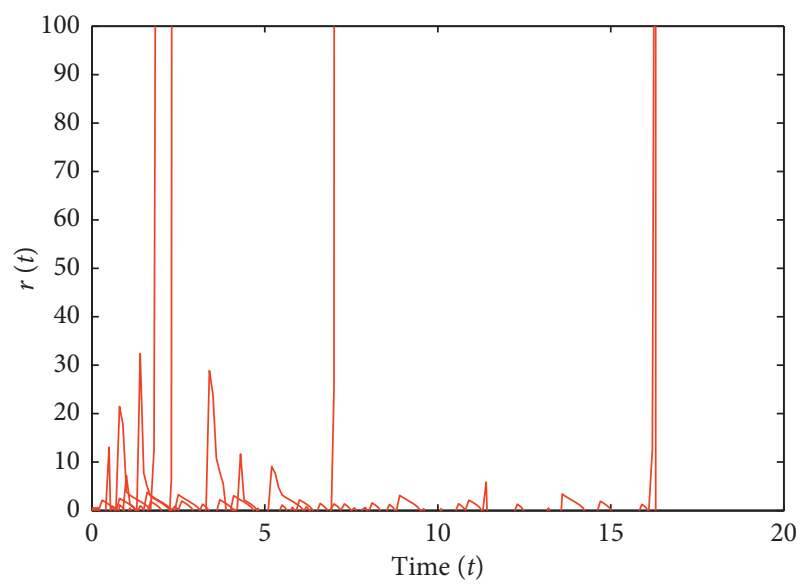

Figure 7: Unstable trajectory of $r(t)$ of (45) with $\theta=0.1, k=1.02$.

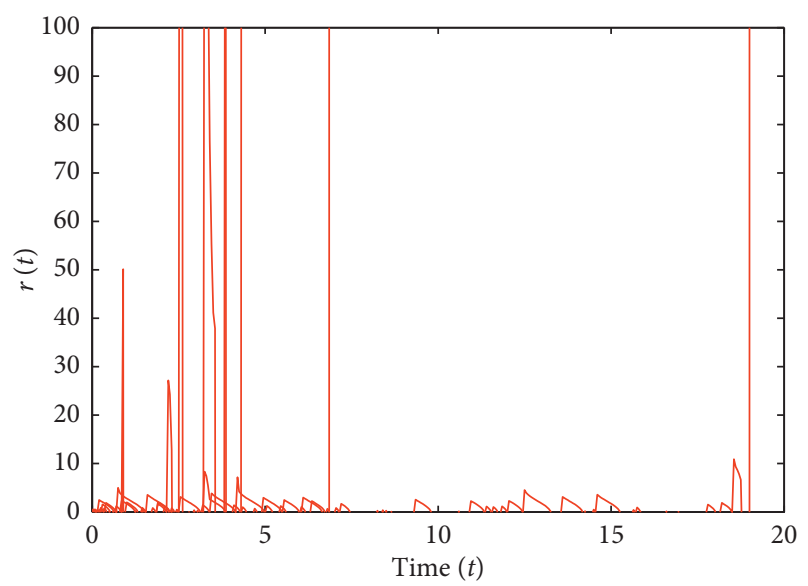

Figure 8: Unstable trajectory of $r(t)$ of (45) with $k=0.01, \theta=0.5$.

In Figure 9, where $k=1.02, \theta=0.5$, which are unsuitable with the conditions in Theorem 1, the state trajectories of NPRNN (45) are unstable.

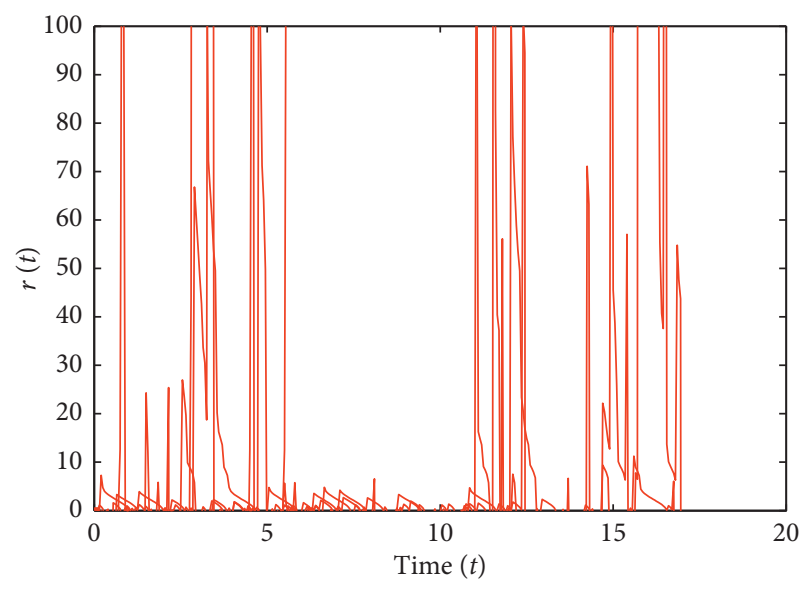

FIGURE 9: Unstable trajectory of $r(t)$ of (45) with $k=1.02, \theta=0.5$.

\section{Conclusion}

Throughout the paper, we mainly analysed the robustness of EGS of NPRNN. Firstly, we provided a leading Lemma 1 to clarify the relationship between $r(\delta(t))$ and $r(t)$. Secondly, we respectively estimated the maximum interval length of the PCAs and the scope of NT compression coefficient that the disturbed NPRNN can tolerate to remain stable. Additionally, we theoretically testified that if the bounds of the interval length of PCAs and NT compression coefficient are all lower than the upper bounds derived herein, NPRNN will still remain stable. In the end, two examples were provided to show that if the real selected values are all lower than the derived values, the system will hold steady. Conversely, as long as one of the two selected values does not satisfy the theoretical result deduced, the system would be destabilized. In other words, we have successfully verified the robustness of the NPRNN model proposed herein. At the same time, we can see from the figures that the trajectory of NPRNN decays exponentially to stability; that is, we also have achieved the robustness of EGS of NPRNN. The criteria provided here are meaningful, which can provide more possibilities for future design and application of NPRNN or any other differential systems with neutral terms and piecewise constant arguments. For instance, future investigations may aim to consider the complex-valued type or fractional-order type of NPRNN with other disturbed factors.

\section{Data Availability}

No data were used to support this study.

\section{Conflicts of Interest}

The authors declare that there are no conflicts of interest regarding the publication of this paper.

\section{Acknowledgments}

The authors thank everyone who provided helpful suggestions for this article. This work was supported by the Natural Science Foundation of China under Grant nos. 62072164 and 12074111 and the Directive Project of Science and 
Technology Research Plan of Hubei Education Department in 2020 under Grant no. B2020127.

\section{References}

[1] J. Cao and J. Wang, "Global asymptotic stability of a general class of recurrent neural networks with time-varying delays," IEEE Transactions on Circuits and Systems I: Fundamental Theory and Applications, vol. 50, no. 1, pp. 34-44, 2003.

[2] C. Vidhya, S. Dharani, and P. Balasubramaniam, "Global asymptotic stability of stochastic reaction-diffusion recurrent neural networks with Markovian jumping parameters and mixed delays," The Journal of Analysis, vol. 27, no. 1, pp. 277-292, 2019.

[3] J. Cao and J. Wang, "Global asymptotic and robust stability of recurrent neural networks with time delays," IEEE Transactions on Circuits and Systems I: Regular Papers, vol. 52, no. 2, pp. 417-426, 2005.

[4] Y. Shen and J. Wang, "Robustness analysis of global exponential stability of recurrent neural networks in the presence of time delays and random disturbances," IEEE Transactions on Neural Networks and Learning Systems, vol. 23, no. 1, pp. 87-96, 2012.

[5] H. W. Zhang, Z. S. Wang, and D. R. Liu, "Robust exponential stability of recurrent neural networks with multiple timevarying delays," IEEE Transactions on Circuits and Systems II: Express Briefs, vol. 54, no. 8, pp. 730-734, 2007.

[6] Z. G. Zeng, J. Wang, and X. X. Liao, "Global exponential stability of a general class of recurrent neural networks with time-varying delays," IEEE Transactions on Circuits and Systems I: Fundamental Theory and Applications, vol. 50, no. 10, pp. 1353-1358, 2003.

[7] L. L. Wang, "Robust multistability and multiperiodicity of neural networks with time delays," Advances in Neural Networks, vol. 9377, pp. 146-153, 2015.

[8] C.-A. Popa and E. Kaslik, "Multistability and multiperiodicity in impulsive hybrid quaternion-valued neural networks with mixed delays," Neural Networks, vol. 99, pp. 1-18, 2018.

[9] L. Duan, Q. Wang, H. Wei, and Z. Y. Wang, "Multi-type synchronization dynamics of delayed reaction-diffusion recurrent neural networks with discontinuous activations," Neurocomputing, vol. 401, pp. 182-192, 2020.

[10] L. Q. Zhou and Z. X. Zhao, "Exponential synchronization and polynomial synchronization of recurrent neural networks with and without proportional delays," Neurocomputing, vol. 372, pp. 109-116, 2020.

[11] C. Vidhya, S. Dharani, and P. Balasubramaniam, "Stability of impulsive stochastic reaction diffusion recurrent neural network," Neural Processing Letters, vol. 51, no. 5, pp. 1049-1060, 2020.

[12] G. Bao, Y. Peng, X. Zhou, and S. Q. Gong, "Region stability and stabilization of recurrent neural network with parameter disturbances," Neural Processing Letters, vol. 52, no. 3, pp. 2175-2188, 2020.

[13] W. T. Chang, S. Zhu, J. Y. Li, and K. L. Sun, "Global MittagLeffler stabilization of fractional-order complex-valued memristive neural networks," Applied Mathematics and Computation, vol. 338, pp. 346-362, 2018.

[14] L.-F. Wang, H. Q. Wu, D.-Y. Liu, D. Boutat, and Y.-M. Chen, "Lur'e Postnikov Lyapunov functional technique to global Mittag-Leffler stability of fractional-order neural networks with piecewise constant argument," Neurocomputing, vol. 302, pp. 23-32, 2018.
[15] X. F. Li, J.-A. Fang, and H. Y. Li, "Exponential adaptive synchronization of stochastic memristive chaotic recurrent neural networks with time-varying delays," Neurocomputing, vol. 267, pp. 396-405, 2017.

[16] X. F. Li, J.-A. Fang, W. B. Zhang, and H. Y. Li, "Finite-time synchronization of fractional-order memristive recurrent neural networks with discontinuous activation functions," Neurocomputing, vol. 316, pp. 284-293, 2018.

[17] C. X. Huang, X. Long, and J. D. Cao, "Stability of antiperiodic recurrent neural networks with multiproportional delays," Mathematical Methods in the Applied Sciences, vol. 43, no. 13, pp. 6039-6102, 2020.

[18] W. L. Jiang, L. L. Li, Z. W. Tu, and Y. M. Feng, "Exponential Lagrange stability for impulses in discrete-time delayed recurrent neural networks," International Journal of Systems Science, vol. 50, no. 1, pp. 50-59, 2019.

[19] A. Bellen, N. Guglielmi, and M. Zennaro, "On the contractivity and asymptotic stability of systems of delay differential equations of neutral Type," BIT Numerical Mathematics, vol. 39, no. 1, pp. 1-24, 1999.

[20] Z. Jackiewicz, "One-step methods of any order for neutral functional differential equations," SIAM Journal on Numerical Analysis, vol. 21, no. 3, pp. 486-511, 1995.

[21] C. J. Zhang and Y. Y. He, "The extended one-leg methods for nonlinear neutral delay-integro-differential equations," $A p$ plied Numerical Mathematics, vol. 59, no. 6, pp. 1409-1418, 2009.

[22] C. Li and C. G. Zhang, "Block boundary value methods applied to functional differential equations with piecewise continuous arguments," Applied Numerical Mathematics, vol. 115, pp. 214-224, 2017.

[23] C. J. Zhang, C. Li, and J. Y. Jiang, "Extended block boundary value methods for neutral equations with piecewise constant argument," Applied Numerical Mathematics, vol. 150, pp. 182-193, 2020.

[24] W. J. Lv, Z. W. Yang, and M. Z. Liu, "Stability of the EulerMaclaurin methods for neutral differential equations with piecewise continuous arguments," Applied Mathematics and Computation, vol. 186, no. 2, pp. 1480-1487, 2007.

[25] W.-S. Wang, S.-F. Li, and K. Su, "Nonlinear stability of RungeKutta methods for neutral delay differential equations," Journal of Computational and Applied Mathematics, vol. 214, no. 1 , pp. $175-185,2008$.

[26] C. J. Zhang, W. S. Wang, B. C. Liu, and T. T. Qin, “A multidomain legendre spectral collocation method for nonlinear neutral equations with piecewise continuous argument," International Journal of Computer Mathematics, vol. 95, no. 12, pp. 2419-2432, 2018.

[27] Y. Shen and J. Wang, "Robustness analysis of global exponential stability of non-linear systems with time delays and neutral terms," IET Control Theory \& Applications, vol. 7, no. 9, pp. 1227-1232, 2013.

[28] C. M. Wu, J. H. Hu, and Y. Li, "Robustness analysis of hybrid stochastic neural networks with neutral terms and timevarying delays," Discrete Dynamics in Nature and Society, vol. 2015, no. 3, pp. 1-12, 2015.

[29] S. R. Grace, J. R. Graef, and I. Jadlovská, “Oscillation criteria for second-order half-linear delay differential equations with mixed neutral terms," Mathematica Slovaca, vol. 69, no. 5, pp. 1117-1126, 2019.

[30] H. Chen and C. Zhu, "Delay-dependent exponential stability for uncertain neutral stochastic linear systems with interval time-varying delay," IET Control Theory \& Applications, vol. 6, no. 15, pp. 2409-2418, 2012. 
[31] S. M. Shah and W. Joseph, "Advanced differential equations with piecewise constant argument deviations," International Journal of Mathematics and Mathematical Sciences, vol. 6, no. 4, pp. 671-703, 1983.

[32] K. L. Cooke and J. Wiener, "Retarded differential equations with piecewise constant delays," Journal of Mathematical Analysis and Applications, vol. 99, no. 1, pp. 265-297, 1984.

[33] M. U. Akhmet, D. Aruğaslan, and E. Yılmaz, "Stability analysis of recurrent neural networks with piecewise constant argument of generalized type," Neural Networks, vol. 23, no. 7, pp. 805-811, 2010.

[34] X. Y. Li, H. X. Li, and B. Y. Wu, "Piecewise reproducing kernel method for linear impulsive delay differential equations with piecewise constant arguments," Applied Mathematics and Computation, vol. 349, pp. 304-313, 2019.

[35] F. Karakoç, "Asymptotic behaviour of a population model with piecewise constant argument," Applied Mathematics Letters, vol. 70, pp. 7-13, 2017.

[36] M. X. Zha, W. X. Si, and T. Xie, "Robust exponential stability of recurrent neural networks with deviating argument and stochastic disturbance," European Journal of Pure and Applied Mathematics, vol. 13, no. 4, pp. 794-806, 2020.

[37] J.-E. Zhang, "Robustness analysis of global exponential stability of nonlinear systems with deviating argument and stochastic disturbance," IEEE Access, vol. 5, pp. 13446-13454, 2017. 